\title{
Image Segmentation Algorithms on MR Brain Images
}

\author{
G. Evelin Suji \\ Department of Electrical and \\ Electronics, \\ BSF Institute of Technology, \\ Bangalore
}

\author{
Y.V.S. Lakshmi, PhD. \\ Manager- IPR, C-DOT, \\ Bangalore
}

\author{
G. Wiselin Jiji, PhD. \\ Department of Computer \\ Science and Engineering, \\ Dr. SivanthiAditanar College of \\ Engineering \\ Tiruchendur- 15,
}

\begin{abstract}
Magnetic Resonance Image plays a major role in medical diagnostics. Image segmentation is done to divide an image into meaningful structures. Image segmentation is the initial step in image analysis and pattern recognition. It becomes more important while dealing with medical images where presurgery and post-surgery decisions are required for the purpose of initiating and speeding up the recovery process. Manual segmentation of abnormal tissues cannot be compared with modern day's high speed computing machines. Segmentation is done to extract the features of the image that are used for analysis, interpretation, and understanding of images. Accuracy of the extracted features decides the accuracy of the algorithm. Selection of a suitable algorithm is highly based on the application. This paper highlights the various image segmentation algorithms, used in medical images.
\end{abstract}

\section{Keywords}

Accuracy; Algorithm; Analysis; Features; Segmentation

\section{INTRODUCTION}

MR brain image consists of four regions namely Gray Matter (GM), White Matter (WM), Cerebrospinal fluid (CSF) and background. The image pixels values can be considered as subsets of parameters including the time constant characterization T1 (magnetization vector along longitudinal axis), T2 (Transverse Component) and proton density. Image segmentation is a difficult task in image processing. Image segmentation divides an image into meaningful structures or objects by locating all pixels or voxels that form its boundary or by identifying them that belong to the object. A pixel in a particular region is similar to the other pixel with respect to some criteria such as color, texture, edge, motion, etc. Segmentation algorithms are area based instead of pixel based.

\section{CHALLENGES OF MR BRAIN IMAGE SEGMENTATION}

In the last two decades, researchers are applying a number of segmentation algorithms to segment medical images. Only few algorithms are suitable for medical image analysis due to complexity and inaccuracy. Moreover, there are no standard image segmentation techniques available to produce satisfactory results for MR Brain images. The challenge of brain image segmentation is the optimal selection of features, tissues, brain and other elements etc. The other challenges include supervision, manual thresholding and verification procedure. Segmentation by the experts is variable [1]. Hence, there is a need for a computer based system that defines the boundaries of brain tissues accurately with minimum user intervention $[2,3]$.

\section{CLASSIFICATION OF IMAGE SEGMENTATION TECHNIQUES}

Image segmentation refers to the process of partitioning an image into groups of pixels or different regions that are homogeneous with respect to certain criterion. Most of the segmentation algorithms are based on the characters of gray level pixels such as discontinuity around edges and similarity in the regions. Segmentation approach can be classified as: 1) Edge based segmentation 2) Region-based segmentation and 3) Theory based segmentation [4] (see Figure 1). These approaches are used for improving the segmentation process and for producing maximum reliability.

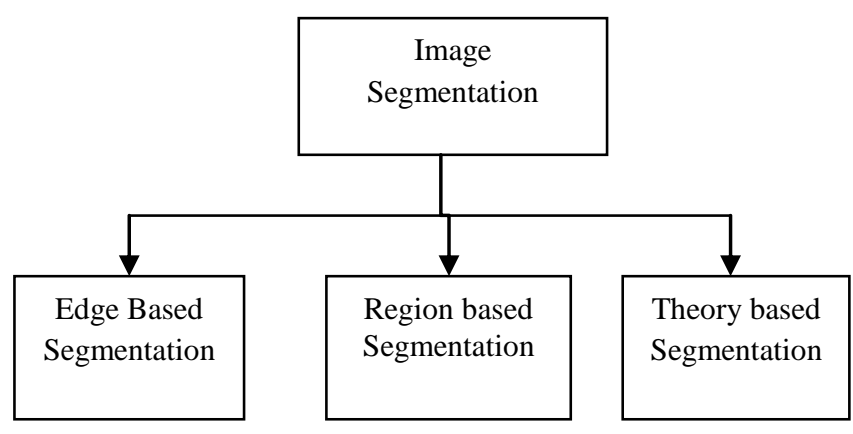

Figure 1: Classifications of Segmentation approaches

\subsection{Edge based Segmentation}

Edge based segmentation algorithm is applied to detect boundaries and discontinuities in an image. The edge is determined by the extreme of the first-order derivative or a zero crossing in the second-order derivative [5]. Edge detection is usually done with linear gradients operators such as Prewitt kernel, Robert Kernel, Sobel Kernel, Frei-chen Edge detector, Laplacian of Gaussian and Canny Edge detecting techniques. These operators work well with sharp edges and less noise $[6,7,8]$. Laplacian of Gaussian and Canny operator are less sensitive to noise as compared to other operators. These techniques are done based on the way that the human perceives object and can produce good result for images having good contrast between regions. Edge based segmentation does not work well with ill-defined edges or with too many edges and less immune to noise [4].

\subsection{Region based Segmentation}

Region based segmentation approach partitions an image into regions that contains connected pixels with similar properties based on a set of predefined criteria. Region based approach is done based on the assumption that neighboring pixels within one region have similar values. Thresholding, region growing, region splitting, region merging and level set approaches falls under this category. The basic operations involved in this 
approach are i) Merging, ii) Splitting

\subsubsection{Region Growing}

Region growing approach is done by grouping the neighboring pixels with similar intensities. This process is iterated for each boundary pixel in the region and requires a seed to begin the iteration. Based on the property defined by the user, this approach separates the region. This approach works with noisy environment and the borders are perfectly thin and connected. The demerits of this approach are: i) The seed point is user dependent ii) The algorithm is highly iterative and time consuming iii) It works with homogeneous area $[6,9,3]$

\subsubsection{Region Splitting and Merging}

Region splitting and merging approach takes the spatial information into consideration and look for uniformity within a sub region based on intensity, color and texture, etc. An image is recursively divided until all regions satisfy a homogeneity criterion. This approach is useful for visualizing a problem and implementing a solution. The algorithm is simple and works well with noisy environment. The region borders are thin and connected. The main advantage of this technique is that the image can be split progressively according to our required resolution. The demerits of this approach are: i) The seed point is user dependent ii) wrong seed point can lead to wrong segmentation result iii) The algorithm is highly iterative and time consuming iv) The segmentation may produce blocky segments as splitting is done in rectangular quadrants. v) This method requires a suitable sub segments to be established before performing splitting and is time consuming $[6,7,8]$.

\subsubsection{Thresholding based Segmentation}

Thresholding approach produces segments having pixels with similar intensities. This technique neglects the spatial information and involves in selecting a single gray level value, Threshold, to divide the image pixels into several classes and there by separating the object from the background. Thresholding based segmentation attempts for finding a threshold value that enables to classify the pixels into different categories. Thresholding approach can be further classified into: i) Global Thresholding ii) Local Thresholding iii) Adaptive Thresholding iv) Histogram based Thresholding. The major advantage of this approach is that it does not require any prior information of the image. This approach is useful for establishing boundaries in images that contain solid objects on contrast background. Thresholding approach has less computational complexities. The approach is simple and effective for images with different intensities. The demerits of this approach are: i) This approach is not suitable for multi-channel images ii) It does not work well for an image having no sharp peaks or with broad and flat valleys iii) Factors such as inadequate contrast, noise, non-stationary objects and ambient illumination can complicate the thresholding operation. [7,8,3]

\subsubsection{Level Set Segmentation}

Level set segmentation is a powerful algorithm used for medical image segmentation. This approach can handle any cavities, convolution, and concavities that are present in images. The initial step in this approach is to specify the curve. The accuracy of the output is based on the position of the curve with respect to the object boundary. This technique provides better output only if the curves are placed near symmetrically with respect to the object boundary [10]. Level Set segmentation approach is a stable approach and easy to compute. This approach can be applied to solve problems involving moving interfaces, singularities geodesics in moving curves, surfaces, and etching etc. [11]. The merits of this technique are: i) stable approach ii) Equations used in this approach are not stiff and Geometric quantities are easy to compute iii) Simplicity in computing three dimensional problems iv) Level set segmentation is useful to solve problems of moving interfaces. The major demerit is that the result is good only if the initial curves specified are placed near symmetrically with respect to the object boundary.

\subsection{Theory Based Segmentation}

Theory based segmentation algorithm are derived from the fields of knowledge such as Fuzzy mathematics, artificial intelligence, genetic algorithm and Neural network etc.

\subsubsection{Fuzzy Approach}

Fuzzy set theory and Fuzzy logic have been applied to handle the uncertainty and ambiguity manifested in images. Quantitative analysis and qualitative evaluation can be done by applying fuzzy pattern recognition method. Fuzzy operators, mathematics and inference rules are used in image segmentation [12, 13, 14, 15, 16]. In Fuzzy set approach, each pixel in an image has a degree to which it belongs to a region or a boundary, characterized by membership value [4]. Fuzzy cognitive map approach is used to model the behavior and operation of complex system. This approach is the fusion of fuzzy logic and cognitive map theories. This technique is mainly used to detect the automatic changes in images. The merits of this approach are: i) Fuzzy approach can represent the relationship between the input pattern data and clusters more naturally ii) Feature selection algorithm based on this approach improves accuracy and suitable for noisy images. iii) Fuzzy approach is used to detect the automatic changes in the images. The demerits of this approach are: i) The iteration is larger and complex ii) Computational complexities of this approach are intensive iii) Fuzzy membership determination is not a trivial job.

\subsubsection{Neural Network Approach}

Neural Networks are basically information processing systems that have certain performance characteristics in common with biological neural network. The image is mapped into a neural network where each neuron represents a pixel. The edge of the image is extracted by using dynamic equations to detect the state of every neuron towards minimum energy defined by neural network $[4,17,18]$. Neural networks have been applied successfully for face detection [7]. The merits of this approach are : i) Fast computational capability ii) The algorithm can establish modeling for any process due to its ability to interact among processing units iii) Insensitive to noise iv) Feasibility of training a system to capture the complex class-conditional density of face pattern. v) Neural network approach produces best result for real-time application. The demerits are: i) Knowledge of one of the segmentation algorithm is required ii) The results of the image segmentation have the influence of initialization iii) The period of training is very long iv) Chances of over training in due process.

\subsubsection{Watershed Transformation}

Watershed transformation is a powerful tool for image segmentation. It can also be classified as region based segmentation. In this approach, the image is considered as a 
topographic surface. The gray level of the image represents the altitude. The region with a constant gray level represents the flat zone of the image. The region edges correspond to high watershed and low gradient region interior corresponds to catchment basin (see Figure 2). By analyzing the intensity in an image as elevation, and simulating rainfall, the image is decomposed into watershed region $[7,8,11]$.

The watershed algorithm works well, if each local minima corresponds to an object of interest. It can extract nearly uniform (blob like) objects from the background. The demerits of this algorithm are over segmentation, sensitive to noise, poor performance in low contrast boundaries and poor detection of thin edges.
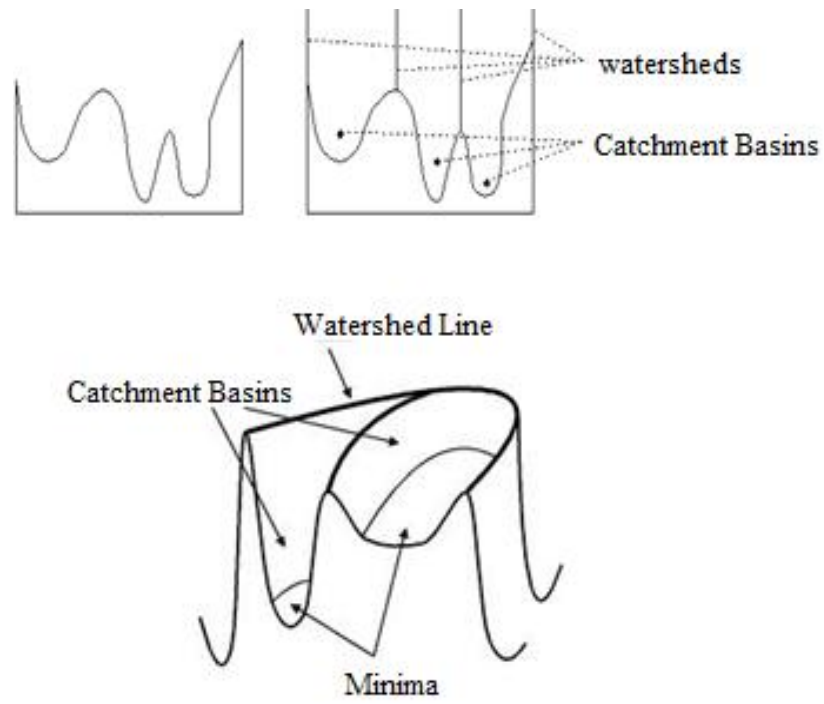

Figure 2: a) Gray level profile of image data b) and c) Watershed Segmentation

\section{CONCLUSION}

Image segmentation is an essential step in analyzing problems and pictorial pattern recognition .Computer vision has find its application in medical, satellite, movement detection and so on. The different segmentation algorithms used for medical imaging have been discussed in this paper. The choice of one segmentation algorithm over the other is based on the particular characteristics of the problem being considered. Further works may be conducted to develop an efficient algorithm for MR Brain Image Segmentation.

\section{REFERENCES}

[1] S. Wareld, J. Dengler, J. Zares, C. Guttmann, W. Gil, J. Ettinger, J. Hiller, and R. Kikinis, Automatic identication of grey matter structures from mri to improve the segmentation of white matter lesions, Journal of Image Guided Surgery, 1(6):326-338,1995.

[2] Mathew C. Clark, Segmentating MRI Volumes of the Brain With Knowledge- Based Clustering. MS Thesis, Department of Computer Science and Engineering, University of South Florida, 1994.
[3] M. Masroor Ahmed, Dzulkifli Bin Mohamad, Segmentation of Brain MR Images for Tumor Extraction by Combining Kmeans Clustering and Perona-Malik Anisotropic Diffusion Model, International Journal of Image Processing, vol.2, issue (1), pp 27-34.

[4] Sameena Banu, The comparative study on color Image segmentation Algorithm, IJERA, vol 2, pp 1277 1281,2012

[5] Krishna Kant Singh, Akansha Singh, A study of Image Segmentation Algorithms For Different Types of Images, International Journal of Computer Science, vol 7, 2010.

[6] Vipula Singh, Digital Image Processing with MATLAB and LabVIEW, Reed Elsevier India Private Limited.

[7] S. Jayaraman, S.Esakkirajan, T. Veerakumar, Digital Image Processing, Tata McGraw Hill Education Private Limited.

[8] R.C Gonzalez, R.E Woods and S.L Eddins, Digital Image Processing Using MATLAB, Pearson, Fifth Impression,2009.

[9] Issac N. Bankman, Hand book of Medical Image Processing and analysis, Second Edition, Accademic press,2008.

[10] Ravikanth Malladi, James A. Sethian and Baba C. Vemuri, Shape modeling with front propagation: a level set approach, IEEE Transactons on Pattern analysis and machine intelligence, vol 17,1995.

[11] D. Narain Ponraj, M. Evangelin Jenifer, P.Poongodi, J. Samuel Manoharan, A survey on the preprocessing Techniques of mammogram for the detection of Breast Cancer,JETCIS, vol2, pp 656-664.

[12] S.K. Pal et al., A review on Image segmentation techniques, Pattern Recognition, 29, 1277,1294, 1993.

[13] J. M. Keller, and C. L. Carpenter, Image Segmentation in the presence of Uncertainty, International Journal of Intelligent Systems, Vol. SMC-15, 193-208, 1990.

[14] S. K. Pal and R. A. King, On Edge Detection of X-ray Images Using Fuzzy Sets, IEEE Transaction on Pattern Analysis and Machine Intelligence, Vol. PAMI-5, No.1, 69-77, 1983.

[15] I. Bloch, Fuzzy Connectivity and Mathematical Morphology, Pattern Recognition letters, Vol. 14, 483488, 1993.

[16] T. L. Huntsberger, C. L. Jacobs and R. L. Canon, Iterative Fuzzy Image Segemntation, Pattern Recognition, Vol. 18, No. 2, 131-138, 1985.

[17] Zhenghao Shi, Lifeng He, Application of neural Networks in Medical Image Processing, Proceedings of the second International Symposium on Networking and Network Security. ISBN 978-952-5726-09-1, 2010, $023-$ 026.

[18] K. Suzuki et al, Neural Edge Enhancement from Noisy Images, IEEE Trans. Pattern Ana. Mach.Intell. 25, 2003 , $1582-1596$ 